\title{
Mortalidad materna en el Instituto Materno Infantil de Santafé de Bogotá D.C. 1985-1989
}

\author{
Pío Iván Gómez Sánchez*; Nicolás Ruiz C.**; Janeth Pulido C.***
}

\begin{abstract}
RESUMEN: En el presente estudio, se revisan 5 años (1985-1989) de mortalidad materna en el Instituto Materno Infantil de Santafé de Bogotá, mostrando: las causas, factores de riesgo, calidad de atención médica e institucional. Se calcula la tasa de mortalidad materna para el período revisado analizando las fluctuaciones de la misma en los últimos $\mathbf{3 0}$ años, teniendo en cuenta que es un indicador fiel de la calidad de atención brindada a la mujer gestante.
\end{abstract}

El tipo de estudio es descriptivo, retrospectivo, teniendo como instrumento de recolección un formulario precodificado que contiene las variables a estudiar.

Se encontraron 113 muertes maternas en este período y 50792 nacidos vivos, dando una tasa de mortalidad no depurada de 22.25 x 10000 nacidos vivos. Hubo 98 muertes de causa directa, 8 indirectas y 7 no maternas. Las tres primeras causas de muerte fueron en su orden: la sepsis $(41.60 \%)$, siendo ocasionada en tres cuartas partes por abortos provocados, seguida de toxemia (36.73\%) y hemorragia (11.22\%).

Se concluye que es necesario mejorar los recursos institucionales, mejorar la capacitación del personal de salud en medicina preventiva, consejería preconcepcional, control prenatal y estudiar a fondo la problemática de la gestación indeseada.

PALABRAS CLAVE: Mortalidad Materna, Instituto Materno Infantil.

SUMMARY: In the present study, five years (1985-1989) of maternal mortality is studied in the "Instituto Materno Infantil de Santafé de Bogotá", showing the causes, risk factors, quality of medical and institutional attention. Maternal Mortality is calculated for the revised period of time, analizing the fluctuations in the past $\mathbf{3 0}$ years, having into account that this is a faithful indicator of the quality of attention given to the pregnant woman.

The type of study is descriptive, retroactive, having a precodificated format, containing the variables to study as an instrument for the needed data.

113 maternal deaths were found in this period and 50792 born alive, giving an average mortality rate of $22.25 \times 10000$ born alive. There were 98 deaths from direct causes, 8 indirect and 7 non-maternal. The first three causes for death were in its order: sepsis (41.60\%), being caused in three quarter parts by provoked abortions, followed by toxemy (36.73\%) and hemorrage (11.22\%).

It is concluded that it is neccesary to improve the institutional resources, improving the training of the personnel in preventative medicine, preconceptual advice, prenatal control and to study deeply the problem of the unwanted gestation.

KEY WORDS: Maternal Mortality - Instituto Materno Infantil.

\section{Introducción}

El Instituto Materno Infantil (IMI), es un centro docenteasistencial de tercer nivel, donde se atienden pacientes de escasos recursos, en las especialidades de Obstetricia,

* Profesor Asistente. Dpto. Ginecología y Obstetricia. Universidad Nacional.

** Gineco-Obstetra. Universidad Nacional.

*** Gineco-Obstetra Universidad Nacional.
Ginecología y Neonatología. En los últimos años, la tendencia de atención se ha derivado en forma importante hacia la gestante de alto riesgo, convirtiéndose el IMI, en centro de referencia no sólo de Santafé de Bogotá, sino de poblaciones de todo el país.

El propósito de este trabajo es revisar la mortalidad materna en el IMI, durante el período comprendido entre el 1 de enero de 1985 y el 31 de diciembre de 1989, con el fin de 
mostrar las causas de muerte materna, los principales factores de riesgo, la calidad de atención médica e institucional, al igual que proponer formas factibles de disminuir, la mortalidad materna evitable en la Institución. Se hará también un análisis de las fluctuaciones que ha tenido la tasa de mortalidad materna en el IMI, en los últimos 30 años, teniendo en cuenta que dicha tasa, es un indicador bastante fiel de la calidad de atención brindada a la mujer gestante en la Institución.

\section{Mortalidad Materna en el IMI}

Las causas de mortalidad materna, varían de un lugar a otro, dependiendo de varios factores como: edad, paridad, calidad de atención obstétrica y nivel socioeconómico de la mujer.

En países desarrollados, se encuentran tasas de mortalidad bajas, no sobrepasando de 5 por 10000 nacidos vivos, con cifras tan bajas como 0.4 por 10000 , para Dinamarca (1). Se atribuye el descenso en las tasas de mortalidad en estos países a: mejor nutrición, mayor cobertura y mejor calidad en los controles prenatales, mayor número de partos intrahospitalarios, uso más racional de la antibióticoterapia, mejoramiento y mayor número de bancos de sangre y disminución de complicaciones sépticas, en lo que ha influido la legalización del aborto. (2-5).

En los países en vía de desarrollo, como Colombia, la mortalidad materna es muy alta comparada con los países industrializados.

En un estudio realizado por la Corporación Centro Regional de Población, en 25 instituciones hospitalarias, que atienden partos en Colombia, se determinó que la tasa de mortalidad materna, era de 23.1 x 10000 nacidos vivos (6).

Todos los estudios al respecto realizados en el país, muestran como causas de muerte materna: sepsis, toxemia y complicaciones hemorrágicas, siendo en su mayoría muertes evitables. (7-17).

En el IMI se viene realizando la revisión de mortalidad materna, desde 1950. La tasa de mortalidad materna en el IMI, tuvo un descenso rápido entre 1950 y 1960 , pasando de $109.6 \times 10000$, a 45.4 x 10000 , disminuyendo lentamente desde este año hasta 1980 , cuando se encontró en 25.4 x 10000. A partir de 1981 y hasta 1984 , se nota que ha vuelto aumentar (Tabla 1).

Tabla 1

TASAS DE MORTALIDAD MATERNA EN EL IMI. $1954-1984$

\begin{tabular}{|ccc|}
\hline A UTOR & AÑos & $\begin{array}{c}\text { TASA POR 10.000 } \\
\text { NACIDOS VIVOS }\end{array}$ \\
\hline Peralta & $1954-1957$ & 60.12 \\
López, Ruíz & $1960-1965$ & 45.40 \\
Rojas y cols & $1966-1970$ & 35.00 \\
Sánchez & $1970-1975$ & 30.47 \\
Muñoz & $1976-1980$ & 25.04 \\
Becerra & $1981-1982$ & 32.64 \\
García & $1983-1984$ & 40.64 \\
Gómez y cols. & $1985-1989$ & 22.25 \\
\hline
\end{tabular}

\section{Objetivos}

1. Determinar la tasa de mortalidad materna en el IMI, durante los años: 1985, 1986, 1987, 1988 y 1989.

2. Determinar las causas principales de muerte materna en el IMI, durante el mismo tiempo, al igual que su evitabilidad.

3. Analizar las fluctuaciones de la tasa de mortalidad en el IMI, en los últimos 30 años.

4. Detectar las fallas en la atención médica e institucional.

5. Establecer una tasa de mortalidad materna depurada para el IMI.

\section{Materiales y métodos}

Este es un estudio descriptivo, retrospectivo, cuyo universo fueron las pacientes obstétricas fallecidas en el IMI, durante el lapso comprendido entre el 1 de enero de 1985 y 31 de diciembre de 1989.

Los datos se obtuvieron de las historias clínicas archivadas en los departamentos de estadística e informes de necropsias del IMI y Hospital San Juan de Dios, así como del libro de la Unidad de Cuidados Intensivos de este último hospital.

El instrumento de recolección utilizado fue un formulario precodificado, con 3 partes:

ı. Identificación de la paciente.

ᄂ Contenido de las variables en estudio.

c. Tomentarios del investigador.

\section{Definic ón de términos y variables}

1. Miserte materna: Fallecimiento de cualquier mujer, por cualquier causa, mientras se encuentre gestante o dentro de cierto período después de la terminación de la gestación, independiente de la duración o sitio de la misma. Este período aprobado por la Federación Internacional de Ginecología y Obstetricia (FIGO), es de 42 días (18).

Se consideran tres grupos de muerte materna (18)

a. Muerte materna directa: (anteriormente mortalidad obstétrica):

Es el fallecimiento resultante de complicaciones obstétricas del embarazo, trabajo de parto, puerperio, intervenciones, omisiones del tratamiento necesario, tratamiento incorrecto, o combinación de estas causas.

b. Muerte materna indirecta: (Anteriormente muerte obstétrica indirecta): es el fallecimiento obstétrico resultante de enfermedad previa o enfermedad desarrollada durante la gestación parto o puerperio, que no es debida directamente a causas obstétricas, sino que es agravada por los efectos funcionales de la gestación.

c. Muerte no materna: (Anteriormente muerte no obstétrica): es el fallecimiento obstétrico, resultante de causas accidentales o circunstanciales, no relacionadas con la gestación o su tratamiento.

2. Tasa de mortalidad materna: Es el número de muertes maternas directas, indirectas y no maternas, por 10000 nacidos vivos en un período determinado.

3. Nacidos vivos: Según la FIGO, es la expulsión o extracción completa del fruto de la gestación, que pese más de 1000 gramos o tenga más de 28 semanas de gestación, o en su defecto mida más de 35 centímetros y que muestre señales de vida después de la separación de la madre.

4. Edad: Años cumplidos de la paciente según registro en la historia clínica. 
5. Procedencia: Lugar en donde vivió la paciente, los últimos meses antes de ingresar al IMI.

6. Remisión: Organismo de salud o persona que envió la paciente al IMI.

7. Gestaciones: Número de gestaciones anotadas en la historia clínica.

8. Edad gestacional: Tiempo en semanas de gestación, al ingreso de la paciente, calculado a partir del primer día de la última menstruación.

9. Riesgo obstétrico: Se refiere a la probabilidad de presentar complicaciones durante la gestación, parto o puerperio, considerándose gestación de bajo riesgo cuando la madre, el feto o recién nacido, tienen pocas posibilidades de presentar complicaciones y de alto riesgo cuando los mismos estén expuestos a mayores peligros en relación con una gestación normal.

10. Causa de muerte: Corresponde a la registrada en la hoja de egreso, con base en el diagnóstico clínico y/o estudio anatomopatológico.

11. Muerte institucional: Es la muerte materna ocurrida en el IMI, después de 24 horas de hospitalización.

12. Muerte no institucional: Es toda muerte materna ocurrida en el IMI, antes de 24 horas de hospitalización, si la paciente ingresó con una complicación debida o no a su gestación.

13. Atención médica: Se refiere a la calidad del manejo dado a cada paciente, en cuanto a diagnóstico, tratamiento y/ o seguimiento por parte del personal médico del IMI. Se subdivide en:

a. Buena: Cuando el diagnóstico, tratamiento y/o seguimiento de la paciente, cumple las normas establecidas por el Departamento de Ginecobstetricia del IMI, acordes con normas internacionalmente aceptadas.

b. Regular: Cuando existe error u omisión en el diagnóstico, tratamiento y/o seguimiento de la paciente, sin que esto incida directamente en el resultado final.

c. Mala: Siempre que se compruebe error u omisión en el diagnóstico, tratamiento y/o seguimiento de la paciente, influyendo desfavorablemente en la evolución del estado clínico.

14. Atención institucional: Se refiere a la disponibilidad de los recursos humanos y físicos necesarios en el diagnóstico, tratamiento, y/o seguimiento de las pacientes que ingresan al IMI, subdividiéndose en:

a. Buena: Cuando la institución cuenta con personal médico y paramédico suficiente, así como disponibilidad de elementos necesarios para un adecuado diagnóstico, tratamiento y/o seguimiento de las pacientes.

b. Regular: Cuando no existen todos los recursos humanos y físicos considerados necesarios para el manejo integral de las pacientes.

c. Mala: Cuando no se cuenta con los medios necesarios para el diagnóstico, tratamiento y/o seguimiento de las pacientes y esta situación incide negativamente en la evolución posterior.

\section{Resultados}

1. Tasa de mortalidad: Durante el período de 5 años comprendidos entre 1985 y 1989, ocurrieron en el IMI 113 fallecimientos de pacientes obstétricas; durante el mismo

período se registraron 50.792 nacidos vivos, dando una tasa de mortalidad materna de $22.25 \times 10000$ nacidos vivos (Tabla 2).

Tabla 2

TASA DE MORTALIDAD MATERNA POR AÑO (POR 10.000 NACIDOS VIVOS)

\begin{tabular}{|lccccccc|}
\hline AÑO & 1985 & 1986 & 1987 & 1988 & 1989 & TOTAI \\
\hline No. MUERTES & 20 & 24 & 21 & 24 & 24 & 113 \\
\hline $\begin{array}{c}\text { NACIDOS } \\
\text { VIVOS }\end{array}$ & 11.117 & 10.509 & 8.515 & 9.775 & 10.21650 .792 \\
\hline TASA & 16.98 & 22.83 & 24.66 & 24.55 & 23.49 & 22.25 \\
\hline
\end{tabular}

2. Causas de mortalidad materna: Teniendo en cuenta la clasificación de la FIGO se encontró, que 98 muertes $(86.72 \%)$, fueron por causa directa; $8(7.08 \%)$ de causa indirecta y $7(6.2 \%)$ muertes no maternas (Figura 1).

Figura 1

CAUSAS DE MORTALIDAD MATERNA

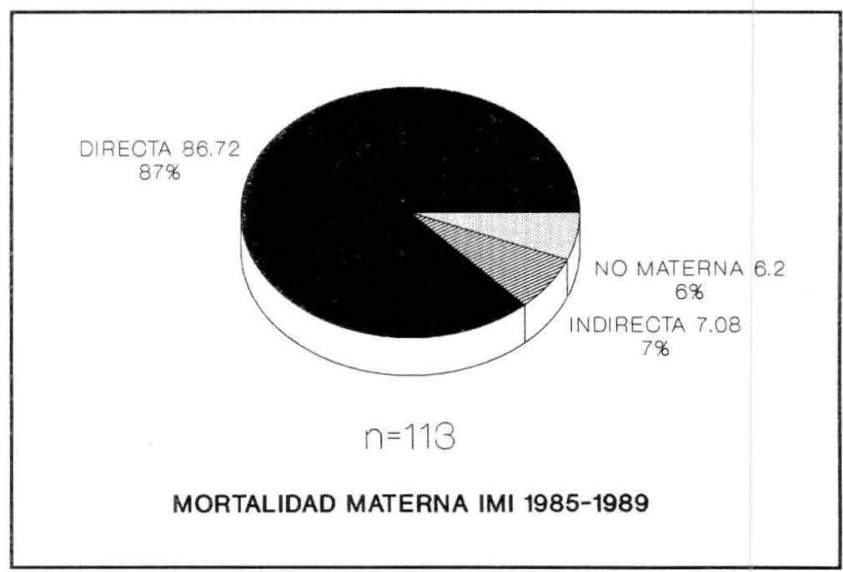

2.1 Muerte materna directa: El mayor número de muertes maternas se debió a complicaciones obstétricas ocurridas

Figura 2

CAUSAS DE MUERTE DIRECTA

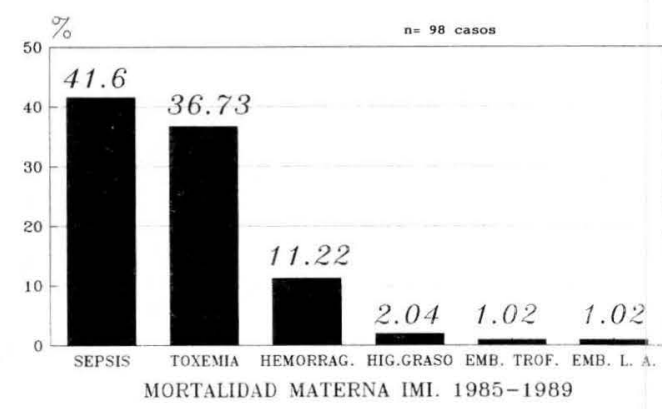


en los diferentes períodos de la gestación y puerperio ( 98 casos). La infección como consecuencia del aborto, parto o cesárea, ocupa el primer lugar de las causas directas (41.60\%), seguida de la toxemia (36.73\%), hemorragia (11.22\%), hígado graso agudo (2.04\%), embolismo trofoblástico (1.02\%) y embolia de líquido amniótico (1.02\%) (Figura 2).

\subsubsection{Mortalidad materna por infección}

Del total de muertes maternas, la principal causa fue la sepsis, encontrándose en este período 47 pacientes $(41.60 \%$ ). En la Figura 3 se presenta la distribución de la infección de acuerdo con el antecedente obstétrico. De las 35 pacientes con aborto, 34 referían maniobras abortivas, aunque algunas muy tardíamente mencionaban este antecedente. De estas, cinco pacientes $(14.28 \%)$ tenían perforación uterina y todas habían consultado por aborto incompleto. De las 4 pacientes puerperas que fallecieron por infección, tres habían tenido el parto fuera del IMI.

Figura 3

MUERTES MATERNAS DIRECTAS POR INFECCION

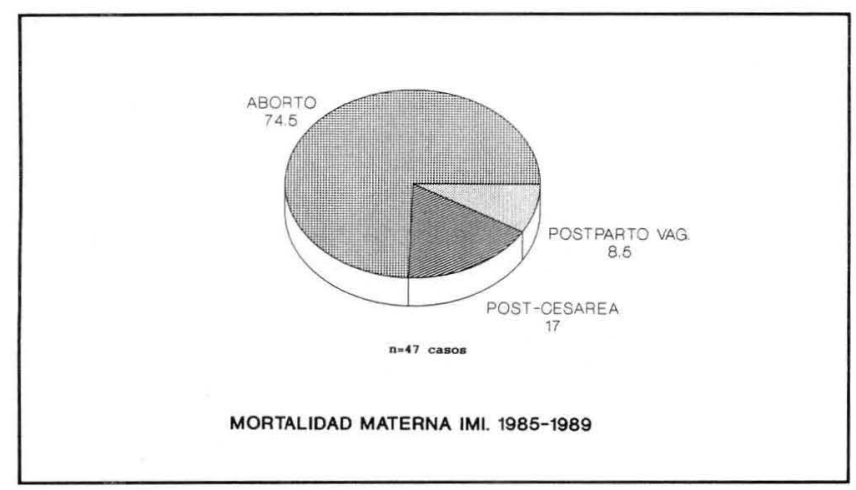

De las ocho pacientes infectadas en el postoperatorio de cesárea, que murieron, la mitad habían sido intervenidas quirúrgicamente fuera del IMI, teniendo todas dehiscencia de la histerorrafia.

Sabiendo que en la mayoría de casos coinciden dos o más estados sépticos, y que en conjunto por sus complicaciones llevan al fallecimiento de la paciente, se investigó la presencia de cada uno de los estados, independientemente de que coincidiera, uno o más de ellos (Figura 4).

Figura 4

MORTALIDAD MATERNA POR INFECCION

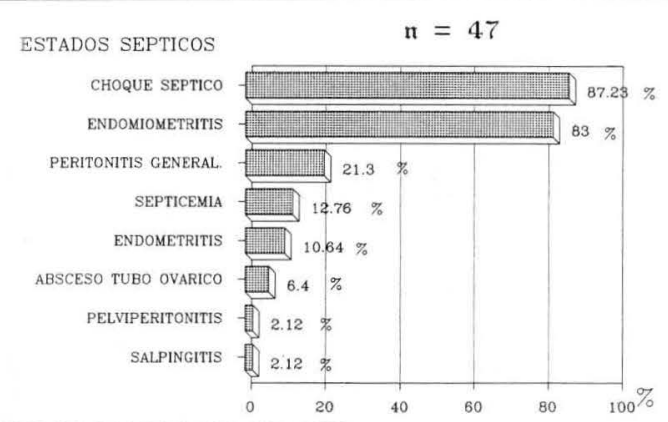

MORTALIDAD MATERNA IMI. 1985-1989
Los estados más frecuentes fueron: el choque séptico en 41 pacientes $(87.23 \%)$, miometritis en $39(82.97 \%)$, peritonitis generalizada en $10(21.27 \%)$, septicemia en $6(12.76 \%)$, absceso tuboovárico en $3(6.38 \%)$, endometritis en 5 (10.6\%) y endosalpingitis aguda en una (2.12\%).

Las complicaciones derivadas de la infección, se presen$\tan$ en la Figura 5.

Figura 5

\section{MORTALIDAD MATERNA DIRECTA POR INFECCION COMPLICACIONES}

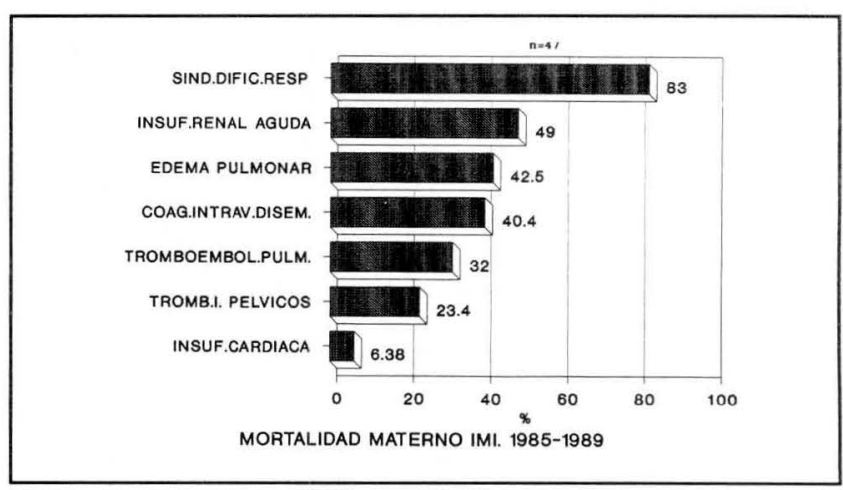

\subsubsection{Mortalidad materna por toxemia}

La toxemia ocupó el segundo lugar como causa de muerte en general; como causa de muerte directa se encontraron 36 casos $(31.85 \%)$, excluyendo las pacientes con antecedentes de enfermedad vascular hipertensiva crónica, teniendo en cuenta solamente los de toxemia pura. Apareció más frecuentemente entre las 24-36 semanas de gestación, presentándose como eclampsia en 24 casos ( $66.6 \%$ ) y como preeclampsia en $12(33.33 \%)$. El momento de aparición fue en preparto en 25 casos $(69.44 \%)$, postparto $7(19.44 \%)$ e intraparto en 4 (11.11\%) (Figura 6).

Figura 6

MUERTE MATERNA DIRECTA POR TOXEMIA

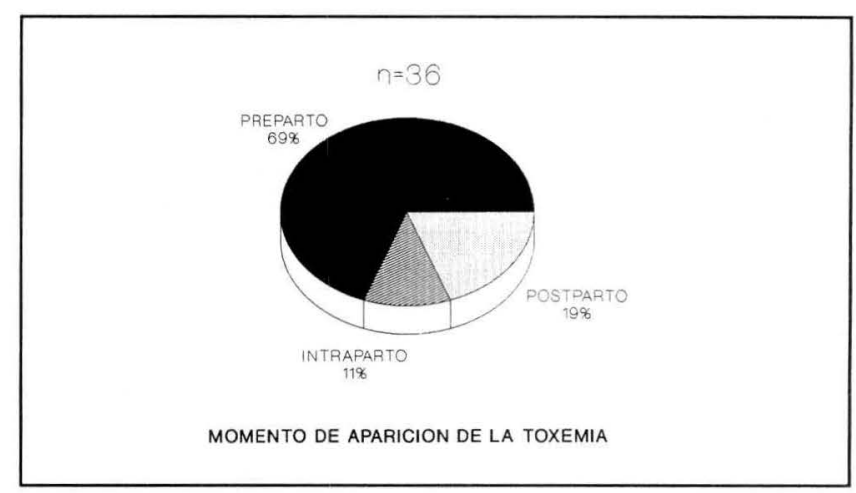

Ocurrieron las siguientes complicaciones: Accidente cerebrovascular en 25 pacientes $(69.44 \%)$, coagulación intravascular diseminada en $9(25 \%)$, edema pulmonar en 13 $(36.11 \%)$, insuficiencia renal aguda en $16(44.44 \%)$, hemorragia hepática en 11 (30.55\%), edema cerebral en $8(22.22 \%)$, hemorragia digestiva alta en $2(5.55 \%)$ e insuficiencia cardíaca congestiva en una paciente $(2.77 \%)$ (Figura 7$)$. 
Figura 7

MORTALIDAD MATERNA DIRECTA POR TOXEMIA COMPLICACIONES

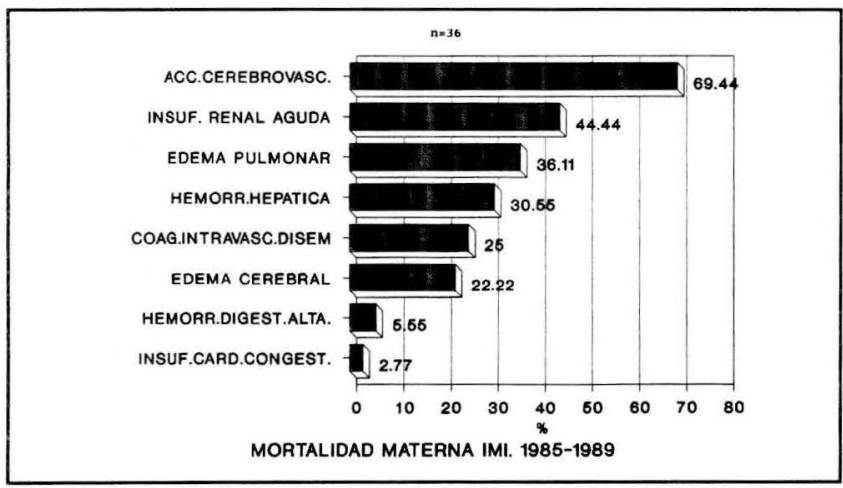

\subsubsection{Mortalidad materna por hemorragia}

Ocupó el tercer lugar como causa de mortalidad materna directa. Las causas de hemorragia se discriminaron así: 5 casos de atonía postparto $(45.45 \%), 3$ ectociesis $(27.27 \%)$, una paciente con coriocarcinoma estado IV sangrante (9.09\%), un caso de acretismo placentario $(9.09 \%)$ y una paciente que presentó Abruptio placentae (9.09\%) (Figura 8).

Figura 8

MORTALIDAD MATERNA POR HEMORRAGIA

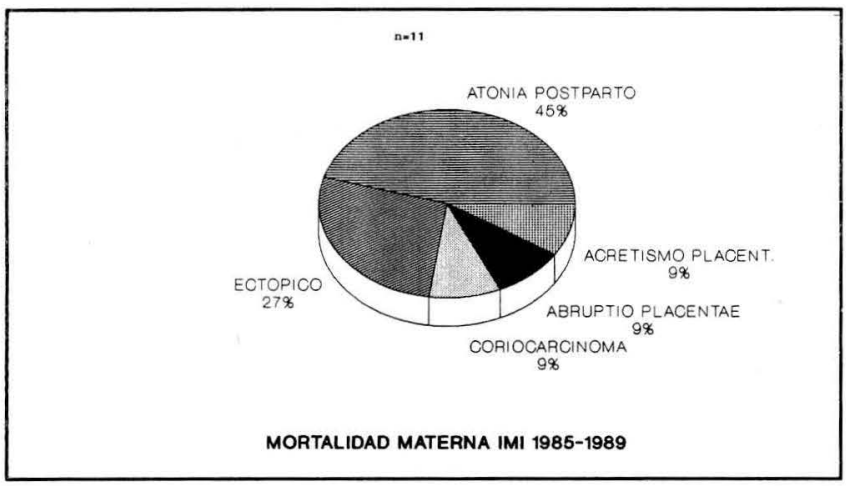

\subsubsection{Otras causas de muerte materna directa}

Se encontraron dos pacientes con hígado graso agudo de la gestación $(2.04 \%)$, una paciente que falleció por embolia de líquido amniótico (1.02\%) y otra por embolismo trofoblástico $(1.02 \%)$.

\subsection{Muerte materna indirecta}

Se estableció la existencia de una enfermedad previa al embarazo, agravada por la gestación en 8 casos, discriminados así:

Tromboembolismo pulmonar en 2 casos (25\%), Cardiopatía en 2 pacientes (25\%) estenosis pulmonar y una insuficiencia mitral), un caso (12.5\%) de aplasia medular, una paciente falleció en una crisis de células falciformes (12.5\%), otra por Hipertensión pulmonar primaria $(12.5 \%)$ y una última con hepatitis viral crónica y pancreatitis hemorrágica (12.5\%) (Figura 9).

\subsection{Muerte no materna}

Se presentaron 7 casos, que equivalen al $6.19 \%$ del total de muertes, distribuidos así: 2 casos $(28.6 \%)$ de Carcinoma
Figura 9

MUERTE MATERNA INDIRECTA

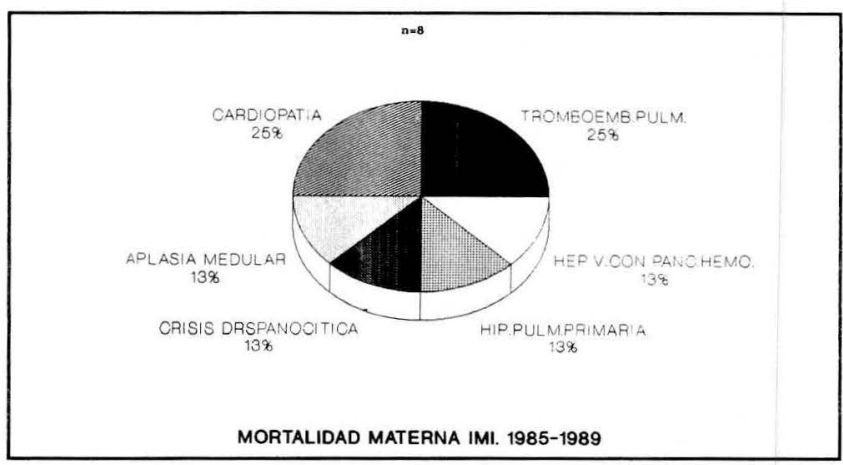

gástrico con carcinomatosis, un caso (14.3\%) de cada una de las siguientes entidades: Bronconeumonía, apendicitis en puerperio inmediato, meningitis purulenta, intoxicación exógena (por fósforo blanco) y un accidente anestésico, ocurrido durante la inducción anestésica de una paciente toxémica, que presentó bloqueo auriculoventricular completo y posterior paro cardiorespiratorio (Figura 10).

Figura 10

\section{MUERTES NO MATERNA}

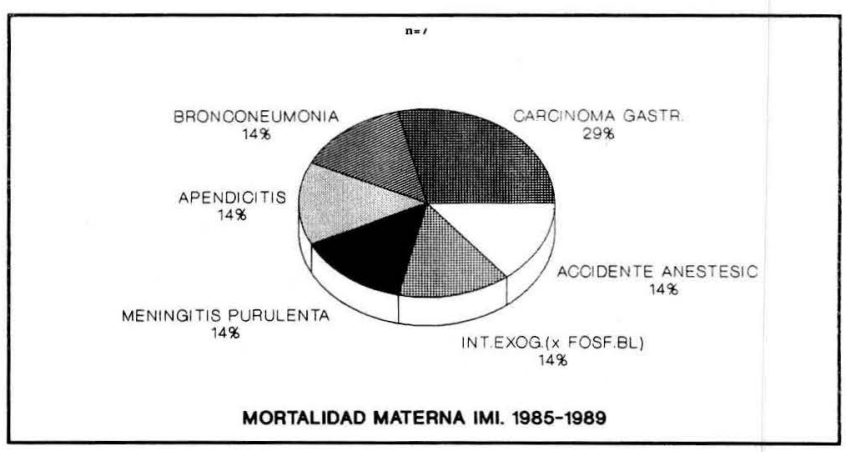

\section{Procedencia y remisión}

En la Figura 11, vemos como el IMI además de brindar atención a la población de la capital de la República, atiende gran parte de Cundinamarca, Boyacá, Tolima y de regiones apartadas como Caquetá y Putumayo, lo que nos muestra, la falta o mala distribución de recursos en esos sitios, determinando gran centralismo y aumento en los costos de atención médica. En cuanto a la remisión, vemos que la mayoría de las pacientes venían de otra institución y tan sólo la quinta parte consultaron directamente al IMI (Figura 12).

Figura 11

LUGAR DE REMISION

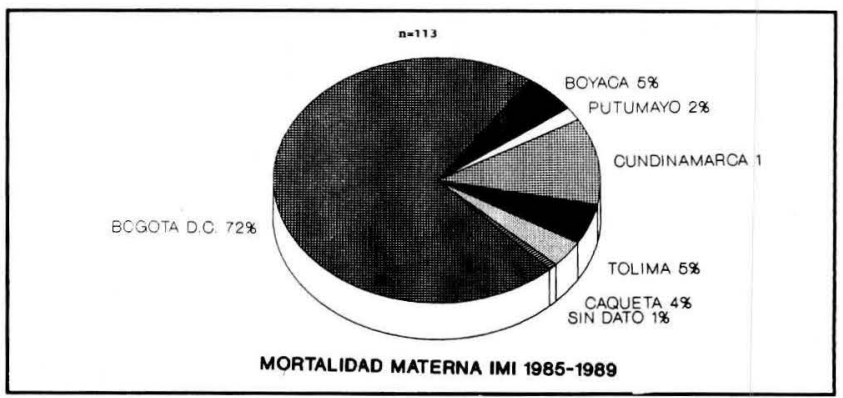


Figura 12

ORGANISMO REMISORIO

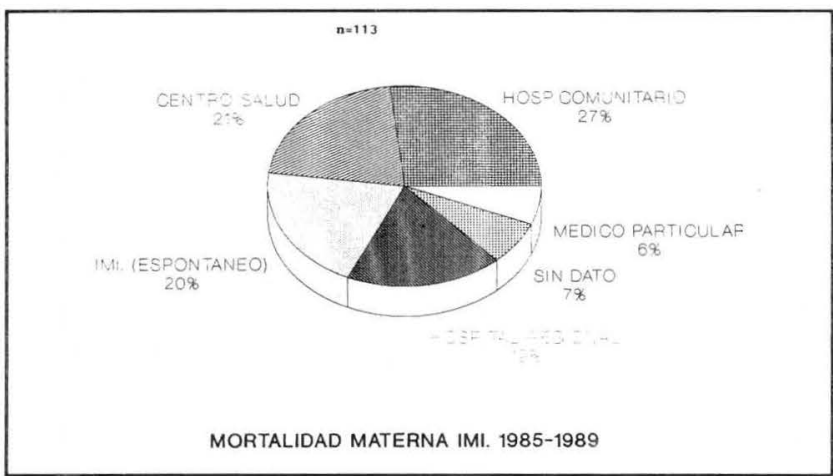

\section{Edad y causa de muerte}

En la Figura 13, vemos como la mayoría de muertes ocurrieron en mujeres del grupo etáreo de 20 a 29 años, con un número importante de fallecimientos en mujeres menores de 19 años.

Figura 13

\section{MORTALIDAD MATERNA DIRECTA E INDIRECTA}

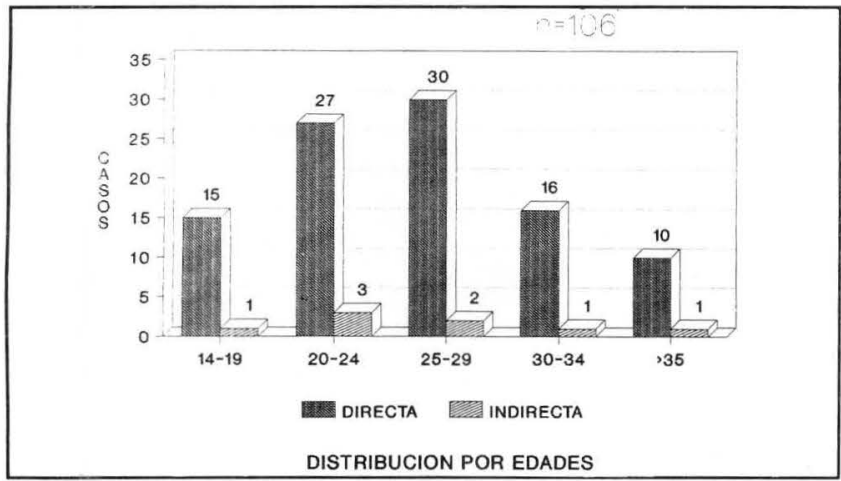

5. Número de gestaciones y causa de muerte

En la Figura 14, se puede apreciar como sigue predominando la mortalidad materna en pacientes primigestantes.

$$
\text { Figura } 14
$$

MORTALIDAD MATERNA DIRECTA E INDIRECTA NUMERO GESTACIONES

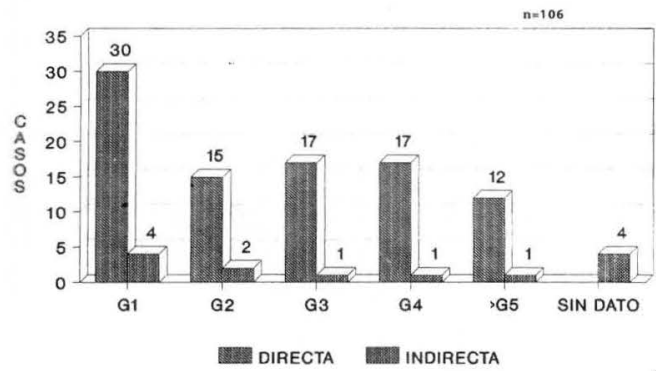

DISTRIBUCION POR NUMERO DE GESTACIONES

\section{Período gestacional}

La mayoría de muertes ocurrieron entre las 30 y 39 semanas de gestación (Figura 15).
Figura 15

MORTALIDAD MATERNA POR EDAD GESTACION

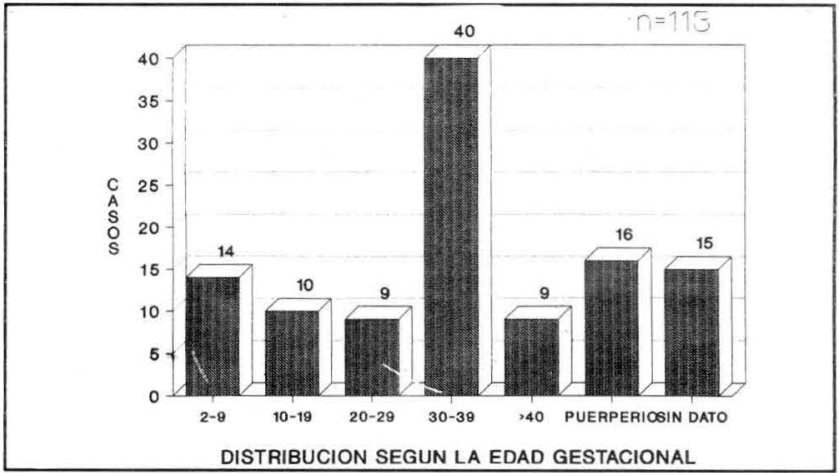

\section{Datos al ingreso}

El $81.41 \%$ de las pacientes no tenían control prenatal. El $70.63 \%$ ingresaron con temperatura normal; $79.64 \%$ estaban conscientes cuando consultaron y $36.28 \%$ tenían cifras tensionales altas (Figura 16).

Figura 16

MORTALIDAD MATERNA DATOS AL INGRESO

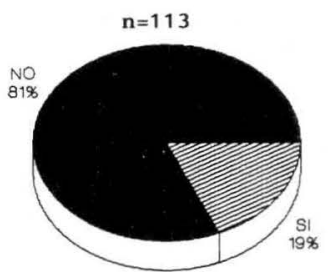

MORTALIDAD MATERNA IML. 1986-1889

MORTALIDAD MATERNA DATOS AL INGRESO ESTADO CONGENGIA

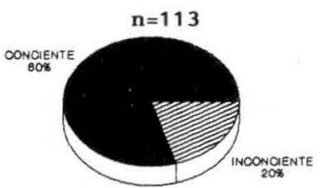

MORTALIDAD MATERNA DATOS AL INGRESO TENSION ARTERIAL

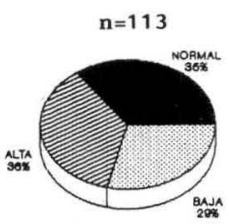

\section{Riesgo obstétrico}

$\mathrm{Al}$ ingreso se consideraron de alto riesgo el $87.61 \%$ de las pacientes, riesgo medio el $10.61 \%$ y bajo riesgo sólo el $1.78 \%$ (Figura 17). 
Figura 17

MORTALIDAI) MATERNA RIESGO OBSTETRICO

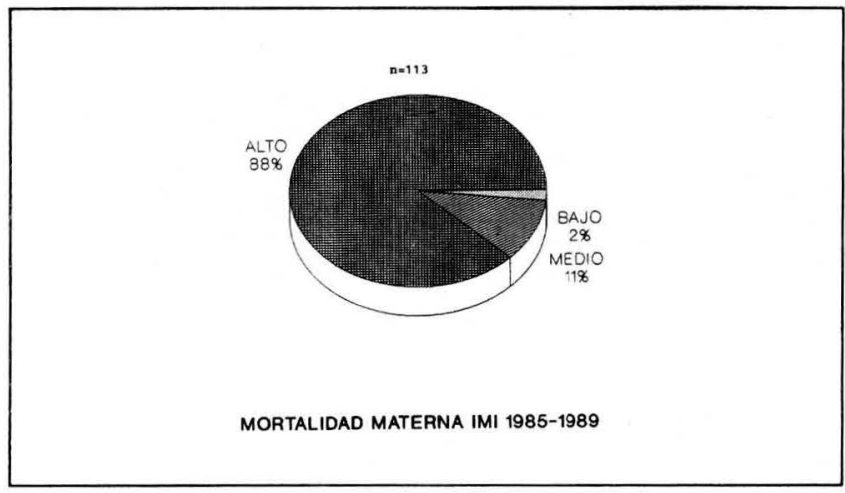

\section{Anatomía patológica}

En un poco más de la mitad de fallecimientos se logró hacer necropsia $(53.09 \%)$ (Figura 18$)$.

\section{Figura 18}

MORTALIDAD MATERNA NECROPSIAS

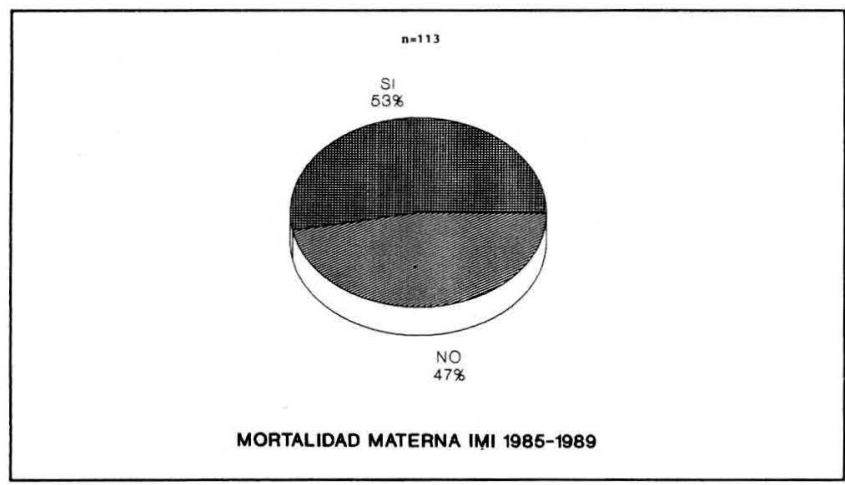

\section{Diagnóstico}

Confrontando el diagnóstico con los hallazgos quirúrgicos, los laboratorios y la anatomía patológica, se evaluó el grado de certeza en el diagnóstico clínico. En los casos en que no coincidió esta afirmación, se consideró errado el diagnóstico y como hay dos momentos en que esto es crucial para el tratamiento, se calificó este error al comienzo y al final de la hospitalización. En este trabajo se encontró que el diagnóstico fue acertado en el $74.33 \%$, errado al principio en el $13.27 \%$ y errado al final en el $4.42 \%$ (Figura 19).

Figura 19

MORTALIDAD MATERNA DIAGNOSTICO

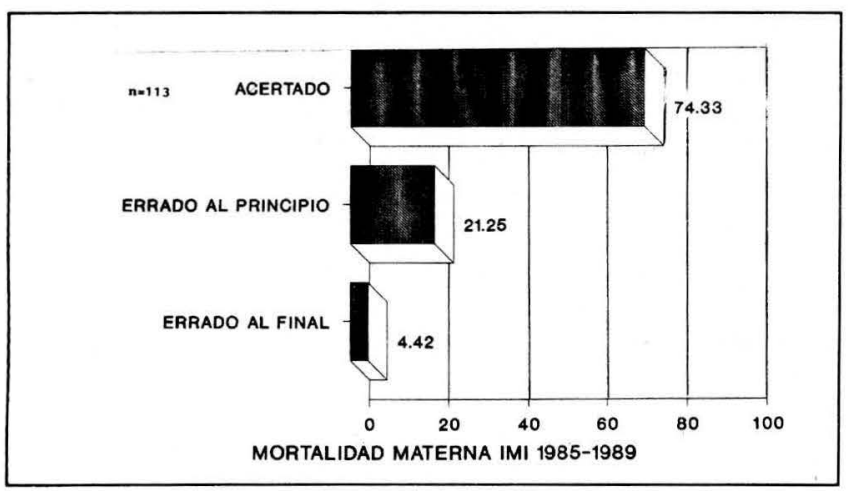

\section{Historia clínica}

La calidad de la historia clínica se consideró buena en el $58.40 \%$, regular en el $21.23 \%$ y mala en el $20.35 \%$ (Figura $20)$.

Figura 20

MORTALIDAD MATERNA HISTORIA CLINICA

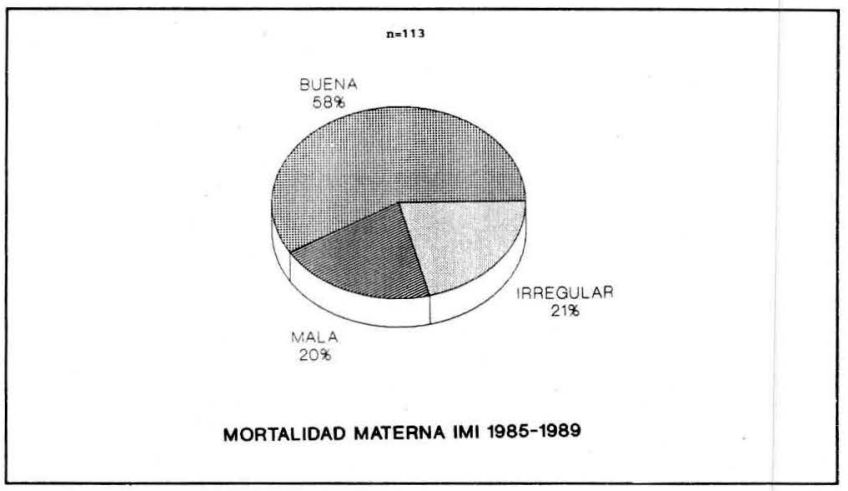

\section{Atención médica}

Se evaluó como buena en el $64.60 \%$ de las pacientes. regular en el $22.12 \%$ y mala en el $13.27 \%$ (Figura 21 ).

$$
\text { Figura } 21
$$

\section{MORTALIDAD MATERNA ATENCION MEDICA}

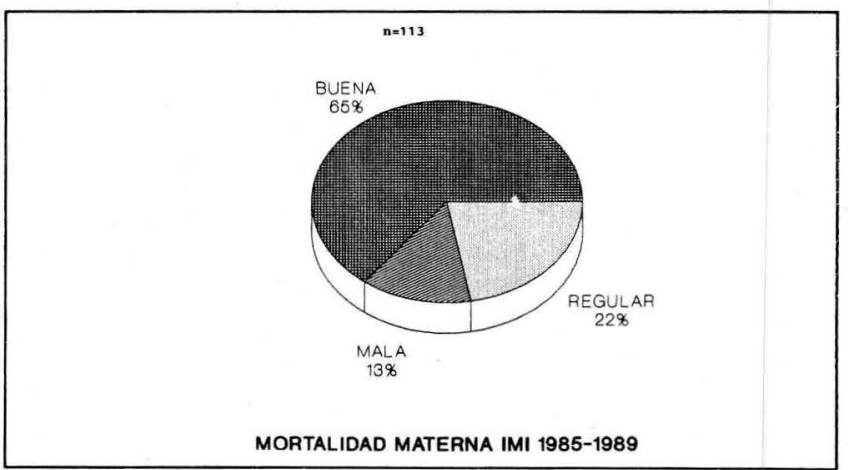

\section{Atención institucional}

Se consideró buena en el 54\%, regular en el $8 \%$, y mala en el $38 \%$, especialmente por la falta de Unidad de Cuidados Intensivos y Banco de Sangre, retardándose o imposibilitándose, la atención inmediata y adecuada de las pacientes (Figura 22).

Figura 22

MORTALIDAD MATERNA ATENCION INSTITUCIONAI

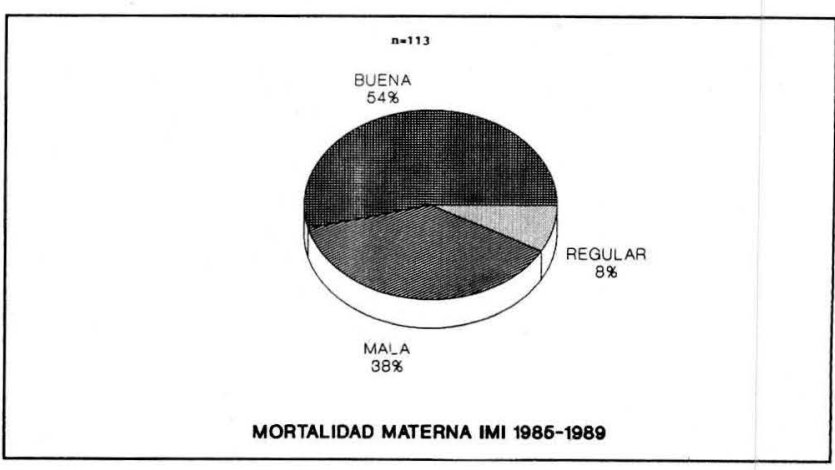




\section{Muerte institucional}

Se encontraron 67 muertes institucionales $(59.3 \%)$ y 46 no institucionales $(40.7 \%)$.

\section{Tasa depurada de mortalidad materna en el IMI}

$\mathrm{Al}$ analizar las historias clínicas, se encontró que en sólo 33 pacientes $(29 \%)$, la muerte institucional se pudo haber evitado, por lo tanto la tasa depurada para el IMI es de 6.49 x 10000 nacidos para el período estudiado, lo cual refleja realmente la calidad de atención en el IMI.

\section{Discusión}

La tasa de mortalidad materna para el IMI, en el período de 5 años revisado (1985-1989), fue de $22.25 \times 10000$ nacidos vivos, que es similar a la encontrada en 25 instituciones del país durante la década pasada (6), (23.1 por 10000 nacidos vivos). Sin embargo al compararla con la tasa para el IMI encontrada en el lustro precedente, hubo disminución (del $37.12 \mathrm{al} 22.25$ ), lo cual corresponde a un descenso del $40 \%$ en la mortalidad materna.

Al igual que en las revisiones de mortalidad en el IMI de los últimos 30 años, (7-17) la principal causa de muerte sigue siendo la directa, con poca variación, encontrándose en este período revisado que el aborto provocado fue la etiología en las tres cuartas partes de los casos sépticos. En países desarrollados, se ha logrado disminuir la morbimortalidad por abortos sépticos, al legalizar el aborto, aumentar la cobertura de los servicios de planificación y llevando educación tanto sexual como contraceptiva a la población. $(3,5)$. En el IMI, se ha logrado reabrir el servicio de planificación familiar desde 1989 , lo cual va a contribuir a disminuir las gestaciones indeseadas y en algún grado el número de abortos provocados. Debe incrementarse la educación contraceptiva y sexual en los establecimientos educativos tanto oficiales como privados y especialmente debemos empezar con nuestros estudiantes del área de la salud a enfocar el aborto no solamente desde el punto de vista médico sino también social, familiar y cultural, para que una vez el profesional de la salud termine su formación académica, tenga capacidad de enfrentar integralmente la problemática de la mujer con gestación indeseada.

Es llamativo como en los últimos dos años revisados, la muerte materna directa por toxemia ha desplazado del primer lugar a la sepsis, que en todos los trabajos precedentes ha sido la primera causa de mortalidad materna. (Tabla 3 ).

Tabla 3

MORTALIDAD MATERNA DIRECTA (1966 - 1989)

\begin{tabular}{|cccc|}
\hline A ÑOS & $\begin{array}{c}\text { INFECCION } \\
(\%)\end{array}$ & $\begin{array}{c}\text { TOXEMIA } \\
(\%)\end{array}$ & $\begin{array}{c}\text { HEMORRAGIA } \\
(\%)\end{array}$ \\
\hline $1966-70$ & 64.00 & 20.50 & 6.60 \\
$1971-75$ & 60.94 & 17.73 & 4.99 \\
$1976-80$ & 69.69 & 19.64 & 7.79 \\
$1981-82$ & 86.20 & 6.90 & 5.20 \\
$1983-84$ & 62.31 & 18.84 & 10.14 \\
$1985-86$ & 58.53 & 29.26 & 7.31 \\
$1987-89$ & 40.40 & 42.10 & 14.00 \\
\hline
\end{tabular}

En los últimos 3 años se nota reducción importante en la sepsis, tendencia que se manifiesta desde 1981, sucediendo lo contrario con la toxemia, que tiende a aumentar progresivamente desde 1983. La mayoría de mujeres con toxemia, no tenían control prenatal y llegaron al IMI en muy mal estado, siendo desde el ingreso el pronóstico malo por la falta de control prenatal adecuado y de remisión oportuna, para prevenir en mayor grado la mortalidad por toxemia.

La muerte por hemorragia sigue ocupando el tercer lugar, incrementándose en los últimos 3 años revisados. Continúa siendo un gran problema la ausencia de Banco de Sangre en el IMI, recomendación dada en trabajos anteriores, así como la creación de una Unidad de Cuidados Intensivos, si tenemos en cuenta el grado de complejidad de las complicaciones con las que ingresan nuestras pacientes.

En cuanto a las muertes directas, casi en su totalidad pudieron ser evitables con consejería antenatal, por lo cual debemos promover no sólo el control prenatal sino la consulta preconcepcional, para detectar los riesgos de una gestación.

Las muertes no maternas son de difícil prevención, pero específicamente las ocasionadas por accidentes anestésicos se pueden evitar, mejorando la tecnología del IMI, con elementos que ayuden a la monitorización permanente de las pacientes (oxímetros, monitores cardíacos, etc).

En cuanto a la remisión, seguimos encontrando que recibimos pacientes de sitios muy lejanos, mostrando mala disposición de los recursos existentes en otros departamentos. Las condiciones de la remisión son muy deficientes, aumentándose el riesgo para la paciente al trasladarla, siendo a veces preferible lograr una adecuada estabilización y posterior remisión. Por lo anterior se debe insistir en la formación universitaria del personal de salud, en instruir sobre las mejores condiciones y formas de remisión cuando se va a derivar un paciente a un nivel de atención superior.

Lastimosamente encontramos que en una institución de tercer nivel como el IMI, a gran parte de las pacientes que fallecen no se les practica necropsia, debiendo ser un requisito para todo fallecimiento en la institución.

Revisando la atención institucional y médica, siguen existiendo los mismos problemas ya identificados en trabajos anteriores como: falta de Unidad de Cuidados Intensivos en el IMI, falta de Banco de Sangre propio, falta de equipos para monitorización y manejo de pacientes críticas (monitores cardíacos, oxímetros, equipos para gasimetría arterial, equipos para soporte ventilatorio, etc). Lo anterior hace que tengamos valores altos de atención institucional inadecuada.

La atención médica se consideró buena en cerca del $65 \%$ de los casos y mala en poco más del $13 \%$, relacionándose con una historia clínica deficiente en la quinta parte de las pacientes, lo cual nos indica la necesidad de crear en el IMI un comité de Historias Clínicas, no sólo para los casos de mortalidad sino que mantenga una alta calidad en la historia de todos los servicios de la institución.

Al calcular la tasa depurada para el IMI encontramos que en el 29\%,"la muerte institucional se pudo haber evitado, con mejores recursos institucionales y mejor atención médica, dando una tasa de mortalidad depurada de 6.49 por 10000 nacidos.

Buscando disminuir la tasa de mortalidad, desde 1989 ha vuelto a funcionar en forma permanente el Comité de Morta- 
lidad y desde 1991, se normatizó la vigilancia del puerperio inmediato en el área de partos, requiriéndose la orden del médico y evolución en la historia, antes de enviar la paciente a la sala de puerperio.

Esperamos lo anterior, junto con mejoramiento de los recursos institucionales, mayor cobertura en planificación familiar e incremento de educación sexual y contraceptiva como programa del Ministerio de Salud, estudio y capacitación por parte del personal de salud en la problemática de la gestación indeseada, así como el concientizar a la comunidad y los proveedores de servicios de salud, de la importancia de los programas de Medicina preventiva, enfatizando en la consejería preconcepcional y control prenatal, sean los pilares para disminuir al máximo la tasa de mortalidad materna, que sigue siendo alta en nuestro país.

\section{BIBLIOGRAFIA}

1. Alvarez B. Mortalidad Materna en el mundo actual. Gaceta Médica Mexicana. 1972; 104: 252-30.

2. Gabel H. Maternal Mortality in South Carolina from 1970-1984, an Analisis. Obstetrics and Gynecology. 1982; 69 (3): 307-311.

3. Requena M. Aborto inducido en Chile. 1a. Ed. The Population Council, Inc. New 1990; 129-178.

4. Sachs, B., Brown D., Driscoll S. Maternal Mortality in Massachussets. Trends and prevention. New England Journal of Medicine. 1987; 316 (11): 667-672.

5. Hani K. y cols. Legal Abortion Mortality inthe United States: 19721982. Am J Obstet and Gynec. 1987; 156: 605-612.

6. Uriza G., López G. Estudio hospitalario de Mortalidad Materna. Rev. Col. Ginecol. 1982; 33 (5): 325-336.

7. Díaz R. Mortalidad Materna en el Hospital Universitario del Valle. Acta Médica del Valle. 1979; 10(3): 112-118.

8. Jubiz y cols. Muertes maternas en el H.S.V.P. Rev. Col. Ginecol. 1978; 29 (4): 178-182.

9. López R. Morbilidad y mortalidad en el IMI. Rev. Col. Ginecol. 1965; 16 (4).
10. López R. Morbilidad y Mortalidad en el Instituto Materno Infantil. Rev. Col. Ginecol. 1968; 19 (4).

11. Peralta y cols. Estudio de Mortalidad en el IMI. Rev. Col Ginecol. 1968; 9:

12. Rojas L. y cols. Mortalidad Materna en el IMI de Bogotá. Rev. Col Ginecol. 1974; 25 (2): 128-149.

13. Sánchez F. Mortalidad Materna en el IMI (1971-1975). Rev. Col Ginecol. 1977; 38: 217-228.

14. SánchezF. Factores que intervienen en la calidad de atención GinecoObstétrica en América Latina.. Rev. Col. Ginecol.1982; 38 (3): 179.

15. Becerra E. Mortalidad Materna en el IMI (1981-1982). Rev. Col. Ginecol. 1986; 37 (5): 328-347.

16. García G., Ñáñez H. Mortalidad Materna en el IMI (1983-1984). Rev. Col. Ginecol. 1986; 37(1): 17-46.

17. Klevens J., Díaz R. Tendencias de la Mortalidad Materna en el IMI. Rev. Col. Ginecol. 1986; 37 (6): 433-442.

18. International Federation of Gynecology and Obstetric. Report on the activity of the Comitte on the Maternal Mortality Journal International Federation Obstetric and Gynecology. 1967; 5: 302. 